\title{
Comité de Morbi-Mortalidad de la Asociación Argentina de Ortopedia y Traumatología
}

\author{
Dr. GERMÁN GARABANO・ggarabano @ gmail.com (1)
}

Cómo citar este artículo: Garabano G. Comité de Morbi-Mortalidad de la Asociación Argentina de Ortopediay Traumatología. Rev Asoc Argent Ortop Traumatol 2018:83(4):228. doi:10.15417/issn. 1852-7434.2018.83.4.910

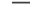

El Comité de Morbi-Mortalidad (CMM) fue creado por la Asociación Argenting

Su origen surge del entendimiento de la AAOT de contar con datos y estadísticas reales sobre el accionar de nuestra especialidad en todo el país. Desde su creación hasta septiembre de este año, fue presidido por la Dra. Lidia Loterzo (a quien agradecemos todo el trabajo y el esfuerzo realizados) y, en la actualidad, está conformado por especialistas de diferentes regiones de nuestro país, con la idea de aumentar el número de integrantes, fomentando una conformación federal.

Se han establecido relaciones con la Federación Argentina de Asociaciones Regionales de Ortopedia y Traumatología para el Ejercicio Profesional (FAAOTEP), la Asociación Argentina para el Estudio de la Cadera y la Rodilla (ACARO), la Asociación Argentina de Trauma Ortopédico (AATO), la Asociación Argentina de Traumatología del Deporte (AATD), con el objetivo de ampliar el marco de su accionar, incentivando el trabajo conjunto. Además, se establecieron relaciones con entidades ajenas a la AAOT, como CESVI (Centro de Experimentación y Seguridad Vial). Y mediante un intenso trabajo con el Ministerio de Salud de la Nación, se logró el acceso a datos de los Egresos Hospitalarios (DEIS), lo cual aporta un flujo muy grande de datos enriquecedores.

El propósito por el cual fue creado es determinar la prevalencia de afecciones ortopédicas y traumatológicas en nuestro país, así como su expresión regional diferenciada, con el objetivo de determinar la Morbi-Mortalidad de diferentes patologías, conocer el resultado de los diferentes tratamientos, así como sus complicaciones, y detallar su correspondiente impacto social. Luego del procesamiento y el análisis epidemiológico de los datos, el comité da a conocer los resultados bajo diferentes modalidades, entre ellas, en el congreso anual de la AAOT, charlas en los diferentes cursos que se dictan en la AAOT, y también en la página web de la entidad madre.

El accionar del comité no termina allí, sino por el contrario, tiene como objetivo brindar a los colegas planes de respuesta para disminuir la Morbi-Mortalidad provocada por las patologías evaluadas.

Para esto, el paso inicial y fundamental es la recolección de datos. Esto se realiza mediante la confección de encuestas, que se encuentran disponibles en la página de la AAOT. Estas han sido confeccionadas junto con la AAOT y las diferentes asociaciones huéspedes involucradas en cada patología. Las encuestas se completan y se envían de manera totalmente anónima bajo la modalidad online.

Actualmente las patologías que se están evaluando son las Fracturas de Cadera y las lesiones asociadas a los Accidentes de Tránsito, y recientemente se sumaron las lesiones del ligamento cruzado anterior, del tendón de Aquiles y las fracturas de húmero pediátricas. La idea de este comité es, con el tiempo, ir sumando aquellas patologías más prevalentes de las diferentes subespecialidades para poder brindar datos reales, actualizados y nacionales, con el propósito de mejorar el accionar traumatológico.

Por último, invitamos a todos los colegas a conocer el comité entrando en la página de AAOT, a leer y completar las mencionadas encuestas con el fin de aumentar la recolección y obtener la mayor cantidad de datos para poder brindar a todos los asociados datos relevantes que constituyan una herramienta útil y necesaria para todos.

\section{Dr. Germán Garabano \\ Presidente}

